\title{
Hydrogen gas post-conditioning alleviates cognitive dysfunction and anxiety-like behavior in a rat model of subarachnoid hemorrhage
}

\author{
JING-HUA SONG, HONG-YAN JIA, TIAN-PENG SHAO, ZHI-BAO LIU and YUAN-PING ZHAO \\ Department of Radioactive Intervention, Cangzhou Central Hospital, Cangzhou, Hebei 061000, P.R. China
}

Received February 23, 2021; Accepted July 19, 2021

DOI: $10.3892 /$ etm.2021.10555

\begin{abstract}
Subarachnoid hemorrhage (SAH) results in high rates of mortality and lasting disability. Hydrogen gas $\left(\mathrm{H}_{2}\right)$ is an antioxidant with demonstrated neuroprotective efficacy. The present study examined the therapeutic efficacy of $\mathrm{H}_{2}$ inhalation on early brain injury following experimental $\mathrm{SAH}$ in rats and the potential underlying molecular mechanisms. The rats were randomly separated into three groups $(n=36$ per group): Sham, $\mathrm{SAH}$ and $\mathrm{SAH}+\mathrm{H}_{2}$. Endovascular perforation of the right internal carotid artery was used to establish SAH. After perforation, rats in the $\mathrm{SAH}+\mathrm{H}_{2}$ group inhaled $2.9 \%$ $\mathrm{H}_{2}$ with regular oxygen for $2 \mathrm{~h}$. Then, $24 \mathrm{~h}$ post-SAH, TUNEL staining was used to detect apoptotic neurons, and both immunostaining and western blotting were conducted to examine changes in p38 MAPK activity and the expression levels of apoptotic regulators (Bcl-2, Bax and cleaved caspase-3) in the ventromedial prefrontal cortex. Then, 30 day post-SAH, Nissl staining was performed to detect neuronal injury, brain MRI was conducted to detect gross changes in brain structure and metabolism, the open field test was used to assess anxiety and the novel object recognition test was performed to assess memory. $\mathrm{H}_{2}$ inhalation following experimental SAH stabilized brain metabolites, improved recognition memory and reduced anxiety-like behavior, the neuronal apoptosis rate, phosphorylated p38 MAPK expression, cleaved caspase-3 expression and the $\mathrm{Bax} / \mathrm{Bcl}-2$ ratio. Collectively, the present results suggested that $\mathrm{H}_{2}$ inhalation can alleviate $\mathrm{SAH}$-induced cognitive impairment, behavioral abnormalities and neuronal apoptosis in rats, possibly via inhibition of the p38 MAPK signal pathway.
\end{abstract}

\section{Introduction}

Subarachnoid hemorrhage (SAH) is a severe type of stroke with high mortality worldwide of 50\% in a 2019 study (1),

Correspondence to: Dr Jing-Hua Song, Department of Radioactive Intervention, Cangzhou Central Hospital, 16 Xinhua Street, Cangzhou, Hebei 061000, P.R. China

E-mail: sjh3711@163.com

Key words: hydrogen gas, apoptosis, subarachnoid hemorrhage, anxiety, cognitive disorder, p38 MAPK and numerous survivors of SAH suffer from long-term disabling sequelae, including sensorimotor, neuropsychiatric and cognitive deficits (2). Several of these impairments can be recapitulated in animal models of SAH, providing opportunities for the elucidation of fundamental pathogenic mechanisms and the identification of potential treatments (3). Depression is a particularly deleterious complication as it impedes rehabilitation and functional recovery following SAH (4). Neuronal apoptosis, oxidative stress and inflammation are seminal pathogenic processes leading to early brain injury and the associated functional deficits after SAH $(2,3,5)$. Multiple cognitive domains are strongly dependent on the prefrontal cortex (PFC), including response inhibition and the encoding of contextual information (6), and inhibition of neuronal apoptosis in the PFC can improve the cognitive performance of SAH model mice (7). A previous study also reported that anxiety behavior was associated with neuronal apoptosis (8). Thus, we hypothesized that cognitive impairment and anxiety behaviors after SAH are associated with neuronal apoptosis in the ventromedial (vm)PFC.

Hydrogen gas $\left(\mathrm{H}_{2}\right)$ administration via inhalation or dissolution in drinking water has been shown to protect neurons in rodent models of traumatic brain injury (9), intracerebral hemorrhage (10) and ischemia-reperfusion injury (11), possibly by suppressing endoplasmic reticulum stress (12), autophagy (13), neuronal apoptosis (14) and/or) inflammatory cell infiltration into cerebral cortex (15). Xie et al (16) reported that $\mathrm{H}_{2}$ provided a neuroprotective effect by alleviating mitochondrial dysfunction via suppressing the nuclear factor erythroid 2-related factor 2-mediated NLR family pyrin domain containing 3 pathway. In addition, hemeoxygenase-1 (HO-1) is involved in $\mathrm{H}_{2}$-mediated neuroprotective effects via the MAPK/HO-1/proliferator-activated receptor $\gamma$ coactivator $1-\alpha$ signaling pathway (17). While the precise molecular mechanisms for these effects remain unknown, recent evidence has suggested modulation of p38 MAPK signaling after SAH exposure may be involved (18).

The MAPK serine/threonine kinases consist of three distinct subtypes, p38 MAPK, JNKs and ERKs 1 and 2, of which p38 MAPK and JNKs are known regulators of apoptosis (19). Activation of p38 MAPK results in the production of cleaved caspase-3, the principal effector of apoptosis, the upregulation of the pro-apoptotic modulator Bax and the downregulation of anti-apoptotic Bcl-2 (20). Inhibition of p38 
MAPK-related proapoptotic pathways can ameliorate early brain injury after experimental SAH (21), and inhibition of p38 MAPK signaling appears to contribute to the neuroprotective effects of $\mathrm{H}_{2}$ (22). Thus, the aim of the current study was to investigate the potential neuroprotective effects of $\mathrm{H}_{2}$ inhalation on neuronal apoptosis, anxiety-like behaviors and cognitive impairments in SAH model rats and to assess the role of the p38 MAPK pathway.

\section{Materials and methods}

Animals. Adult male Sprague-Dawley rats $(\mathrm{n}=108$; age, 6-8 weeks; weight, 200-250 g) were purchased from Liaoning Changsheng Biotechnology and raised under controlled temperature $\left(25 \pm 1^{\circ} \mathrm{C}\right)$, humidity $(50 \pm 10 \%)$ and a $12 / 12$-h light/dark cycle with free access to food and water. All study procedures and animal care protocols were approved by the Animal Review Board of Cangzhou Central Hospital (Cangzhou, China) and conformed to National Institutes of Health guidelines (23).

SAH model and $\mathrm{H}_{2}$ administration. A total of 108 rats were divided into a sham group $(n=36)$, a SAH group $(n=36)$ and a $\mathrm{SAH}+\mathrm{H}_{2}$ group $(\mathrm{n}=36)$. A SAH model was established via the endovascular perforation method as described previously (23). Compared with using two injections of arterial blood solvate into the cisterna magna, a rat model of endovascular perforation was performed in this current study based on the good consistency with the pathophysiological process of SAH (23). Briefly, rats were anesthetized with sevoflurane (7-8\% for induction, 3-4\% for maintenance), intubated and ventilated at a tidal volume of 4-5 ml/1,000 $\mathrm{g}$ body weight (fraction of inspired oxygen $=40 \%$ ) using a ventilator (ALC-V; Shanghai Alcott Biological Technology, Inc.) with continuous body temperature control at $36.0 \pm 0.5^{\circ} \mathrm{C}$ using a heating pad. The oxygen concentration was set to $40 \%$ during surgical preparation and SAH, as $40 \%$ of oxygen could lead to $99-100 \%$ oxygen saturation during mechanical ventilation (24).

Following exposure of the surgical field via lateral neck incision, the blood flow of the common carotid artery (CCA) was blocked using a vascular clamp. A polytetrafluoroethylene tube was then guided up the right internal carotid artery (ICA) through the external carotid artery stump until resistance was detected. A 2-mm protrusion of a tungsten wire inside the tube was used to puncture the intracranial segment of the ICA. After retracting the wire, the CCA was reopened to allow reperfusion via the ICA, and then the external carotid artery was ligatured using a silk thread. At last, the wounds were sutured layer-by-layer. The sham group was subjected to the same procedure but without ICA puncture. In the SAH $+\mathrm{H}_{2}$ group, rats inhaled $2.9 \% \mathrm{H}_{2}$ (25) (Gilmore Liquid Air Company) mixed with $20 \%$ oxygen (flow rate $11 /$ min, oxygen saturation 96-99\%) for $2 \mathrm{~h}$ under anesthesia (2-3\% sevoflurane) immediately after SAH was successfully established. Due to oxidative stress induced by high concentration of oxygen (compared with $40 \%$ oxygen during surgery), 20\% oxygen was used to avoid producing an effect against neuroprotection of $\mathrm{H}_{2}$. The $\mathrm{H}_{2}$ concentration was monitored periodically using a handheld hydrogen detector $\left(\mathrm{H}_{2}\right.$ scan).
Western blotting $(n=6)$ and immunofluorescence assays $(\mathrm{n}=6)$ were performed at $24 \mathrm{~h}$ after SAH, and an MRI study was held at $7(n=6)$ and 30 days $(n=6)$ after SAH surgery. In addition, 30 day post-surgery, Nissl staining $(n=6)$ and behavioral tests $(n=6)$ were conducted. After anesthesia with $8 \%$ sevoflurane, cerebral tissue for western blotting (perfusion with $0.9 \%$ ice-saline via ventriculus sinister), immunofluorescence and Nissl staining assays (perfusion with $0.9 \%$ ice-saline and $10 \%$ neutral-buffered formalin via ventriculus sinister) was extracted. Moreover, once MRI and behavioral tests were finished, the rats were euthanized via cervical dislocation under $8 \%$ sevoflurane anesthesia. The duration of this experiment was 30 days as indicated in Fig. 1. If rats were still alive after 30 days, euthanasia was performed as aforementioned.

Assessment of mortality and $S A H$ grade. The mortality rate of rats was observed within $24 \mathrm{~h}$ after SAH, and the death was verified by the lack of cardiac electric activity. The SAH grade was determined using a previously published grade scale (26). Scores ranging from 0-18, including spontaneous movement of four limbs (0-3), spontaneous activity (0-3), forelimbs outstretching (0-3), body proprioception (0-3), vibrissa touch (0-3) and climbing capacity (0-3), represented the severity of SAH. SAH model rats were divided into three categories according to the severity of bleeding: Mild (SAH grade from 0-7), moderate (SAH grade from 8-12) and severe (SAH grade from 13-18) (27). Once rats were unable to eat food or drink water, euthanasia was performed. Sham-operated rats had a score of 0 . The grading, health and behavior of rats were examined by a blinded observer every $1 \mathrm{~h}$ within $24 \mathrm{~h}$, and every day from $24 \mathrm{~h}$ to 30 days after SAH. The rats with SAH grades $\geq 8$ were included in the following study. The rats with mild SAH were euthanized via cervical dislocation.

MRI study. At 7 and 30 days post-surgery, randomly chosen rats from each group were anesthetized with sodium pentobarbital $(65 \mathrm{mg} / \mathrm{kg})(28)$ and the gross brain structure evaluated using a 3.0 T MRI scanner equipped with a special coil (DISCOVERY MR750; Cytiva). As described in a previous study (29), a predetermined central voxel was used for registration of diffusion-weighted images. Conventional coronal T2-weighted (T2W) images were obtained with the following parameters: Repetition time (TR)/echo time (TE), 3,500/85 msec; number of excitations (NEX), 2; phase, 256; frequency, 320/sec; slices, 21; slice thickness, $1.5 \mathrm{~mm}$; field of view, $80 \mathrm{~mm}$; and acquisition time, $2 \mathrm{~min}$. Then, 7 days post-SAH, cerebral blood flow (CBF) was assessed using $\mathrm{CBF}$-arterial spin labeled (ASL), as the vasospasm was most severe at 7 day after SAH. An axial pseudocontinuous 3D ASL sequence was also performed using the following parameters: TE, $11.1 \mathrm{msec}$; TR, 4,326 msec; field of view, 240x240 mm; and section thickness, $3 \mathrm{~mm}$, spiral readout. Maps of $\mathrm{CBF}$ were generated using Functool software v. 4.5.3 (Cytiva). ${ }^{1} \mathrm{H}$-magnetic resonance spectra were also obtained via multivoxel pattern analysis with the following parameters: TR/TE, 1,500/35 msec; NEX, 1; phase, 18; frequency, 16/sec; and acquisition time, $7 \mathrm{~min} 20 \mathrm{sec}$.

The ventromedial PFC (vmPFC) in T2W and spectral images were further analyzed for infarcts by calculating the ratio of average signal intensity (SI) relative to the 

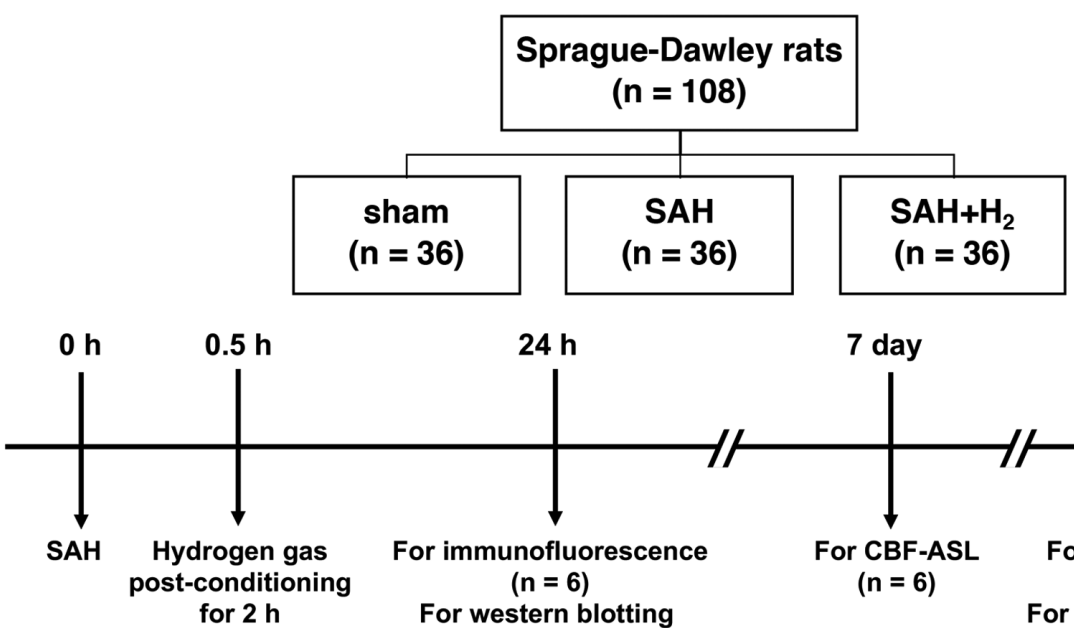

$(n=6)$

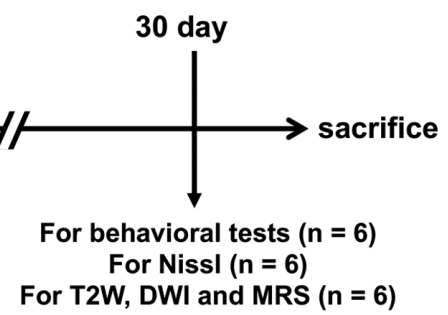

Figure 1. Schematic diagram of the experimental procedures. Rats were subjected to $\mathrm{SAH}$ using a model of endovascular perforation and inhaled $2.9 \% \mathrm{H}_{2}$ mixed with $40 \%$ oxygen and balanced nitrogen (flow rate $1 \mathrm{l} / \mathrm{min}$ ) for $2 \mathrm{~h}$ post-SAH. MRI, including CBF-ASL, T2W, DWI and $1 \mathrm{H}$ MR spectra, pathology and protein expression analyses, and behavior tests including the open field, novel object recognition, were evaluated. SAH, subarachnoid hemorrhage; CBF-ASL, cerebral blood flow arterial spin labeling; T2W, T2-weighted; DWI, diffusion weighted imaging.

temporalis [T2W standardized SI (SSI)]. In addition, the ratio of $\mathrm{N}$-acetylaspartate (NAA) to creatinine $(\mathrm{Cr})$ peak area was calculated to assess neuronal metabolism and integrity using the AW 4.5 Workstation (Cytiva). The positions of NAA and $\mathrm{Cr}$ (as an internal spectral reference) on the nuclear spectrum were 2.02 and 3.05 part per million, respectively.

Open field test (OFT). Considering the motor deficits between rats, long-term anxiety was assessed using an OFT, which was performed at 30 days after SAH (30). The experimental apparatus was a large open field box $(60 \times 60 \times 40 \mathrm{~cm})$ divided into 16 equal squares. The test was conducted 30 day post-SAH. An individual rat was placed in the center of the box, and the distance traveled, grooming and rearing times, and time spent in the corners were recorded over a $90-\mathrm{sec}$ test period and analyzed with a computerized tracking system (XR-XZ301; Shanghai Xinruan Software Co., Ltd.).

Novel object recognition (NOR). The NOR test (30) was conducted in an empty white box $(60 \times 60 \times 40 \mathrm{~cm})$. During the adaptation phase, individual rats were permitted to freely explore the box for $5 \mathrm{~min} / \mathrm{day}$ for 2 consecutive days. In the familiarization phase, individual rats were placed in the same box with two identical objects (tasteless, not smooth) placed in the left and right corners ( $5 \mathrm{~cm}$ from the walls) and allowed to freely investigate both until they had explored each for a total of $30 \mathrm{sec}$ (defined as contact by the front paws or nose). The testing phase was conducted after $24 \mathrm{~h}$. Each rat was placed in the same box with one of the two familiar objects replaced by a novel object. At this stage, continuous monitoring was conducted for $10 \mathrm{~min}$ and preference for the novel object relative to the familiar object was calculated as an index of recognition memory according to the equation: Recognition index $(\mathrm{RI})=$ Novel object exploration time/(Novel object exploration time + Familiar object exploration time).

$H \& E$ and Nissl staining. At 30 days post-SAH, pathological changes in the vmPFC were evaluated via $\mathrm{H} \& \mathrm{E}$ and Nissl staining of coronal brain slices. Briefly, brains were fixed with $10 \%$ neutral-buffered formalin for $48 \mathrm{~h}$ at room temperature, embedded in paraffin, slices at $4 \mu \mathrm{m}$, deparaffinized for $10 \mathrm{~min}$ and were stained with hematoxylin for $5 \mathrm{~min}$ and eosin for $10 \mathrm{sec}$ at $25^{\circ} \mathrm{C}$. For Nissl, the slices were stained as described (C0117; Beyotime Institute of Biotechnology) for $10 \mathrm{~min}$ at room temperature. As Nissl bodies are indicative of protein synthesis, reduced numbers are a sign of nerve cell injury (31). Image-Pro Plus 6.0 (Media Cybernetics, Inc.) was used to count and analyze the number of Nissl-stained cells in six randomly selected fields per animal at magnification of x200 under a light microscope (BX51; Olympus Corporation).

Immunofluorescence. At $24 \mathrm{~h}$ post-surgery, the vmPFC was fixed with $10 \%$ neutral-buffered formalin at room temperature for $48 \mathrm{~h}$, embedding in paraffin, sectioned at $4-\mu \mathrm{m}$ thickness, dewaxed in xylene, hydrated in gradient ethanol at room temperature and heated in sodium citrate for $20 \mathrm{~min}$ at $100^{\circ} \mathrm{C}$. Slices were then incubated overnight at $4^{\circ} \mathrm{C}$ with polyclonal rabbit anti-rat p38 MAPK antibody (1:100; cat. no. AF5887; Beyotime Institute of Biotechnology) and polyclonal mouse anti-rat-neuronal nuclei (NeuN; 1:200; cat. no. ab104224; Abcam). Slices were washed three times with PBS and incubated with $\mathrm{Cy} 3$-conjugated goat anti-rabbit IgG (1:500; cat. no. A0516; Beyotime Institute of Biotechnology) and FITC-conjugated goat anti-mouse IgG (1:500; cat. no. A0568; Beyotime Institute of Biotechnology) for $1 \mathrm{~h}$ at $25^{\circ} \mathrm{C}$. Finally, cell nuclei were counterstained with $5 \mu \mathrm{g} / \mathrm{ml}$ DAPI (cat. no. P0131; Beyotime Institute of Biotechnology) for $3 \mathrm{~min}$ at $25^{\circ} \mathrm{C}$. A fluorescence microscope (MF43; Guangzhou Micro-shot Technology Co., Ltd.) was used to observe the sections.

TUNEL staining was used to identify apoptotic neurons. Slices prepared as described above were dewaxed, washed three times in PBS and incubated in $20 \mu \mathrm{g} / \mathrm{ml}$ protease $\mathrm{K}$ (cat. no. st533; Beyotime Institute of Biotechnology) at $37^{\circ} \mathrm{C}$ for $35 \mathrm{~min}$. Then, the slices were incubated with polyclonal mouse 
anti-rat NeuN (1:200; cat. no. ab104224; Abcam) overnight at $4^{\circ} \mathrm{C}$, following by washing with PBS and incubation with TDT enzyme containing fluorescent labeling solution (cat. no. C1088; Beyotime Institute of Biotechnology) in the dark for $60 \mathrm{~min}$ at $37^{\circ} \mathrm{C}$. TUNEL-stained slices were then treated with $5 \mu \mathrm{g} / \mathrm{ml}$ Antifade Mounting Medium with DAPI (cat. no. P0131; Beyotime Institute of Biotechnology) for $3 \mathrm{~min}$ at $25^{\circ} \mathrm{C}$. Apoptotic neurons were identified by overlapping TUNEL, NeuN and DAPI staining. A fluorescence microscope (MF43; Guangzhou Micro-shot Technology Co., Ltd.) was used to count stained (apoptotic) cells in six fields (magnification, x200) of six sections randomly selected from each rat. The average density of apoptotic cells was determined using Image-pro plus 6.0 (Media Cybernetics, Inc.).

Western blotting. At $24 \mathrm{~h}$ post-surgery, total protein from the vmPFC was extracted in lysis buffer (cat. no. P0013; Beyotime Institute of Biotechnology), and the concentration measured using a BCA Protein Assay kit (cat. no. P0012S; Beyotime Institute of Biotechnology). Equal amounts of protein per gel lane $(30 \mu \mathrm{g})$ were separated using $10 \%$ polyacrylamide gels and transferred to PVDF membranes. Membranes were blocked in QuickBlock ${ }^{\mathrm{TM}}$ Blocking Buffer (Beyotime Institute of Biotechnology) at $25^{\circ} \mathrm{C}$ for $10 \mathrm{~min}$, and probed overnight at $4^{\circ} \mathrm{C}$ with rabbit anti-rat Bax antibody (1:500; cat. no. AB016; Beyotime Institute of Biotechnology), rabbit anti-Bcl-2 antibody (1:500; cat. no. K003505P; Beijing Solarbio Science \& Technology Co., Ltd.), anti-caspase-3 antibody (1:1,000; cat. no. ab13847; Abcam), rabbit anti-rat phosphorylated (p)-p38 MAPK antibody (1:1,000; cat. no. AF5887; Beyotime Institute of Biotechnology), rabbit anti-p38 MAPK antibody (1:1,000; cat. no. AF7668; Beyotime Institute of Biotechnology), rabbit anti-p-JNK1/2 (1:1,000; cat. no. AF1762; Beyotime Institute of Biotechnology), rabbit anti-JNK1/2 (1:1,000; cat. no. AF1048; Beyotime Institute of Biotechnology), rabbit anti-p-AKT (1:500; cat. no. AA329; Beyotime Institute of Biotechnology), rabbit anti-AKT (1:500; cat. no. AA326; Beyotime Institute of Biotechnology) and anti-GAPDH (1:1,000; cat. no. K106389P; Beijing Solarbio Science \& Technology Co., Ltd.), which was used as the loading control. Blotted membranes were then incubated in horseradish peroxidase-labeled goat anti-rabbit secondary antibodies (1:1,000; cat. no. A0208; Beyotime Institute of Biotechnology) at room temperature for $1 \mathrm{~h}$. After three washes in TBS-Tween-20 (0.05\%; cat. no. ST825; Beyotime Institute of Biotechnology), slices were incubated with ECL reagent (cat. no. P0018FM; Beyotime Institute of Biotechnology) for $5 \mathrm{~min}$ at room temperature, and protein bands detected using a western blot detection system (Gel Doc XRS; Bio-Rad Laboratories, Inc.) and quantified by Image Lab software 6.0.1 (Bio-Rad Laboratories, Inc.).

Statistical analysis. All statistical analyses were conducted using SPSS 20.0 (IBM Corp). Group data are presented as the mean \pm SD. The log-rank test of Kaplan-Meier analysis followed by a Bonferroni's test for correction $(\mathrm{K}=3)$ was performed to assess the percentage survival of SAH model rats. In the Kaplan-Meier analysis, if a P-value was less than the Bonferroni-corrected threshold $(\mathrm{P}<0.016)$, then the comparison can be said to be statistically significant. A
Mann-Whitney U test was used to assess the difference of $\mathrm{SAH}$ grade between groups. The Levene test was conducted to check the assumption of homogeneity of variance. Statistical differences among groups were then evaluated via one-way ANOVA followed by Bonferroni's post hoc tests for pair-wise comparisons. $\mathrm{P}<0.05$ (two-tailed) was considered to indicate a statistically significant difference for all tests.

\section{Results}

$\mathrm{H}_{2}$ post-conditioning mitigates the neuroimaging manifestations of SAH-induced neural damage. In total, 21 rats died before the scheduled euthanasia time. None of them were sham-operated animal (0 of 36 rats). The mortality in the SAH group was $26.2 \%$ (16 of 61 rats; sham vs. $\mathrm{SAH}, \mathrm{P}<0.016$; Fig. 2A), which was consistent with previous study (32). However, the mortality dropped to $10.2 \%$ (5 of 49 rats) in the $\mathrm{SAH}+\mathrm{H}_{2}$ group compared with the SAH group ( $\mathrm{P}>0.016$; Fig. 2A). The death of rat all occurred with $5 \mathrm{~h}$ after $\mathrm{SAH}$, and the cause of death was cerebral hernia induced by bleeding volume. In total, 17 rats with mild SAH were excluded from this study. The rats with moderate and severe SAH were included for the following studies. There was no difference in the average $\mathrm{SAH}$ grades between the $\mathrm{SAH}$ and $\mathrm{SAH}+\mathrm{H}_{2}$ groups. ( $\mathrm{P}>0.05$; Fig. 2B).

At 7 days post-SAH, the absolute CBF-ASL map (Fig. 2C) demonstrated that the vmPFC perfusion in SAH and SAH + $\mathrm{H}_{2}$ groups was significantly reduced (vs. sham, $\mathrm{P}<0.05$; Fig. 2D). vmPFC perfusion between both the SAH and SAH + $\mathrm{H}_{2}$ groups did not show significant difference, indicating that $\mathrm{H}_{2}$ inhalation did not improve vasospasm.

At 30 days post-surgery, T2W MRI revealed no significant differences in SSI ratios among sham, $\mathrm{SAH}$ and $\mathrm{SAH}+\mathrm{H}_{2}$ groups (P>0.05; Fig. 2E and F). However, ${ }^{1} \mathrm{H}$ MRS at 30 days post-surgery revealed a significant decrease in the vmPFC NAA/Cr metabolite ratio among SAH group rats compared with sham group rats, which was partially reversed by $\mathrm{H}_{2}$ inhalation ( $\mathrm{P}<0.05$; Fig. $2 \mathrm{E}$ and $\mathrm{G})$. The result indicated the signal intensity of diffusion weighted imaging in the vmPFC was increased in the $\mathrm{SAH}$ and $\mathrm{SAH}+\mathrm{H}_{2}$ groups, but compared with SAH group, the signal intensity was decreased in SAH + $\mathrm{H}_{2}$ group $(\mathrm{P}<0.05$; Fig. 2E and $\mathrm{H})$.

$\mathrm{H}_{2}$ inhalation alleviates anxiety-like behavior following $\mathrm{SAH}$. The OFT is a well-established method to measure anxiety-like behavior in rodents (33). At 30 days post-surgery, SAH group rats showed reduced total travel distance, spent more time in the corners of the open field and less time in the center region, with fewer grooming times and fewer rearings compared with sham group rats $(\mathrm{P}<0.05$; Fig. 3A-E), which are all behavioral signs of anxiety. By contrast, all of these indices of anxiety were partially reversed by $\mathrm{H}_{2}$ inhalation ( $\mathrm{SAH}+\mathrm{H}_{2}$ vs. $\mathrm{SAH}$; $\mathrm{P}<0.05$; Fig. 3).

$\mathrm{H}_{2}$ alleviates cognitive impairment following $\mathrm{SAH}$. The NOR test exploits the innate tendency of animals to preferentially explore a novel object over a familiar object (34). In the familiarization phase conducted 30 days post-surgery, the total time spent in proximity of the two identical objects did not differ, yielding a RI of $\sim 50 \%$ in all groups (P>0.05; Fig. $3 \mathrm{~F}$ and $\mathrm{G}$ ). In 
A

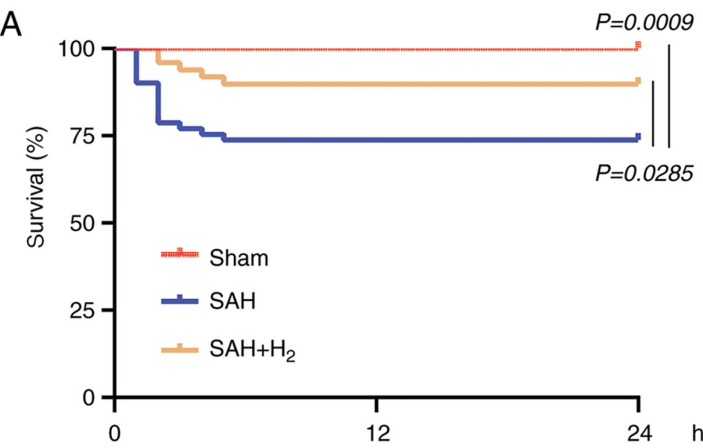

C

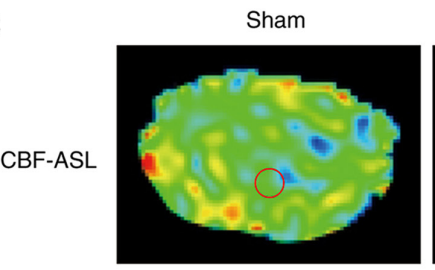

D
B

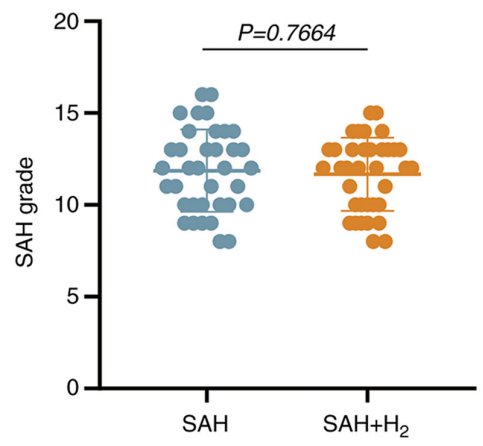

SAH
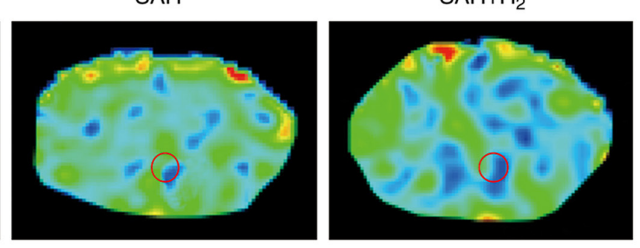

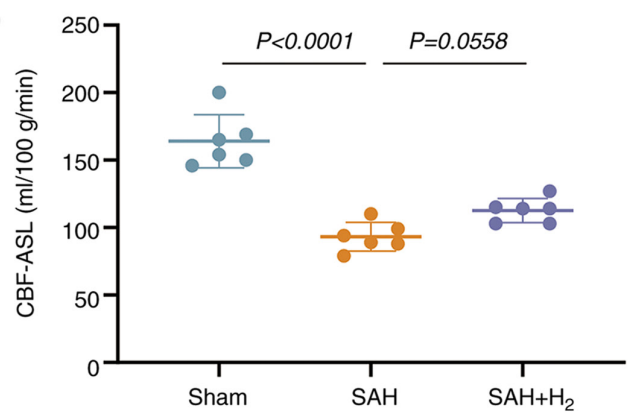

E

T2W1

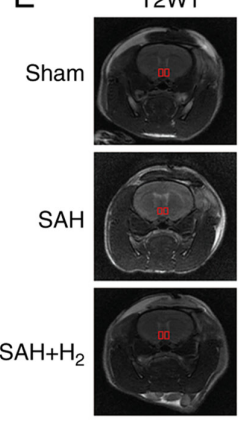

MRS

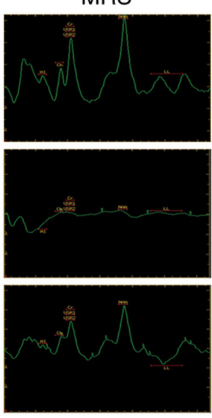

DWI

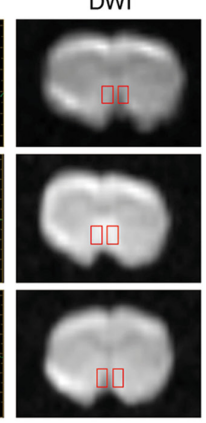

$\mathrm{F}$

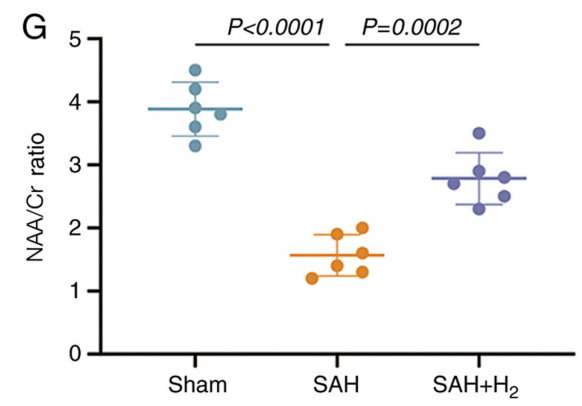

Figure $2 . \mathrm{H}_{2}$ post-conditioning mitigates the neuroimaging manifestations of SAH-induced neuronal damages. (A) Survival analysis during $24 \mathrm{~h}$ after SAH ( $n=36$ in sham group, $n=61$ in $\mathrm{SAH}$ group, $\mathrm{n}=49$ in $\mathrm{SAH}+\mathrm{H}_{2}$ group). (B) $\mathrm{SAH}$ grade of the $\mathrm{SAH}$ group ( $\left.\mathrm{n}=36\right)$ and $\mathrm{SAH}+\mathrm{H}{ }_{2}$ group ( $\mathrm{n}=36$ ). (C) $\mathrm{CBF}-\mathrm{ASL}$ coronal views at 7 day post-SAH. (D) CBF-ASL value in the region of the vmPFC caused by the indicated stimuli. (E) Representative T2W MRI, $1 \mathrm{H}$ MR spectra and DWI of the vmPFC in the coronal view at 30 day post-SAH $(n=6)$. The (F) ratio of standardized signal intensity, (G) the ratio of NAA/Cr and $(\mathrm{H})$ the signal intensity of DWI in the region of the vmPFC caused by the indicated stimuli. SAH, subarachnoid hemorrhage; CBF-ASL, cerebral blood flow arterial spin labeling; NAA, N-acetylaspartate; Cr, creatinine; T2W, T2-weighted; DWI, diffusion weighted imaging; vmPFC, ventromedial prefrontal cortex.

the testing phase, however, SAH group rats spent significantly less time in the defined zone surrounding the novel object compared with sham group rats $(\mathrm{P}<0.05$; Fig. $3 \mathrm{~F}$ and $\mathrm{H})$, and spent more time in the zone surrounding the familiar object, indicating a deficit in recognition memory (lower RI). Inhalation of $\mathrm{H}_{2}$ following SAH significantly increased the RI 
A
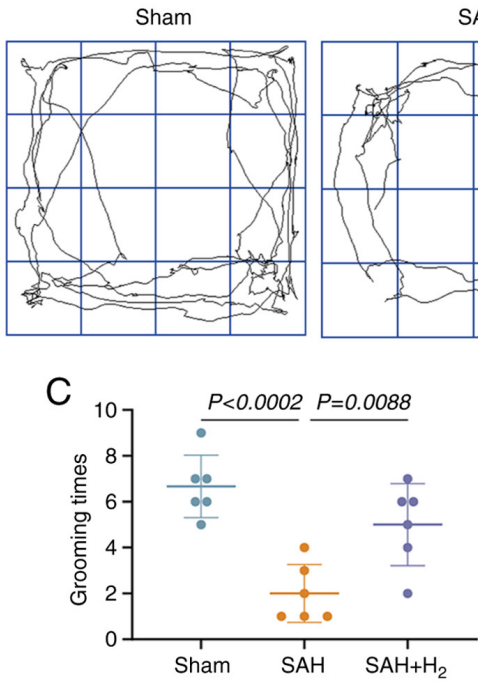

$\mathrm{SAH}$

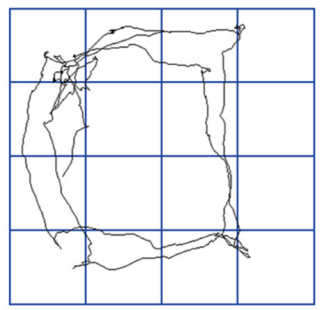

D
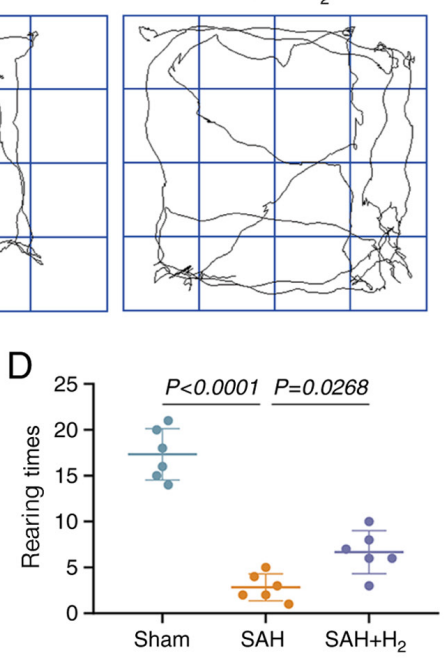

B

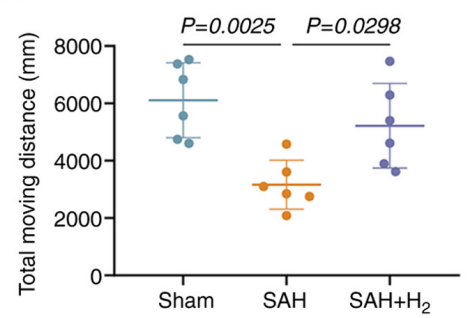

E

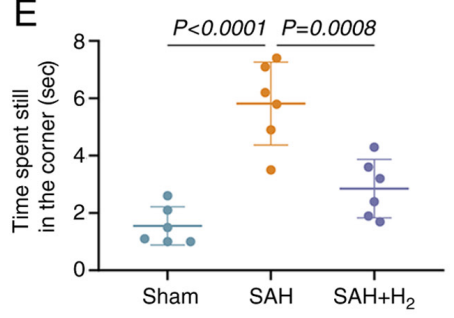

$\mathrm{F}$
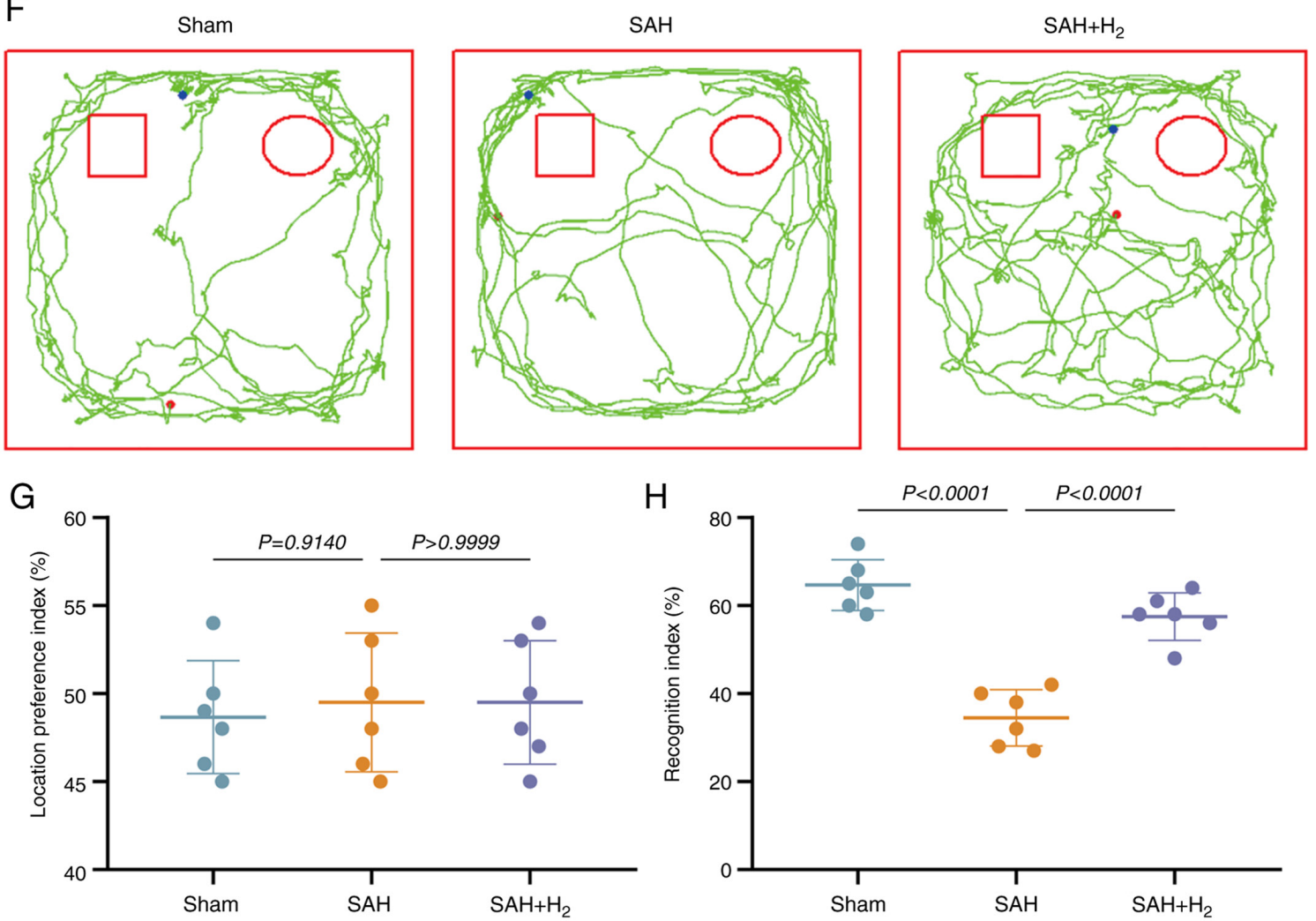

Figure 3. $\mathrm{H}_{2}$ post-conditioning alleviates anxiety-like behavior and cognitive impairment following SAH. (A) Computer printouts showing the shifting trajectories in the OFT caused by the indicated stimuli at 30 day post-SAH. (B) Total moving distance. (C) Grooming times. (D) Rearing times. (E) Time spent still in the corner. (F) Computer printouts showing the shifting trajectories in the NOR caused by the indicated stimuli at 30 day post-SAH. (G) Location preference index. $(\mathrm{H})$ Recognition index $(n=6)$. OFT, open field test; SAH, subarachnoid hemorrhage; NOR, Novel Object Recognition; vmPFC, ventromedial prefrontal cortex.

compared with SAH group rats, indicating partial preservation of recognition memory $(\mathrm{P}<0.05$; Fig. $3 \mathrm{H})$.

$\mathrm{H}_{2}$ inhalation reduces neuronal damage following $\mathrm{SAH}$. Nissl staining of vmPFC tissue from sham group rats revealed structurally intact neurons with the expected somal distribution and abundant Nissl bodies, while vmPFC neurons in the SAH group were disorganized, reduced in number and contained fewer Nissl bodies (vs. sham; $\mathrm{P}<0.05$; Fig. 4A and B). These signs of neurodegeneration were partially reversed by $\mathrm{H}_{2}$ inhalation following $\mathrm{SAH}(\mathrm{P}<0.05$; Fig. $4 \mathrm{~A}$ and $\mathrm{B})$. In addition, H\&E staining was performed to assess the effect of $\mathrm{H}_{2}$ administration, and the data revealed that $\mathrm{H}_{2}$ administration alleviated histological impairments in the vmPFC induced by SAH exposure (Fig. 4C).

$\mathrm{H}_{2}$ reduces neuronal apoptosis following $\mathrm{SAH}$. To investigate the neuroprotective efficacy of $\mathrm{H}_{2}$ inhalation following $\mathrm{SAH}$, neuronal apoptosis in the vmPFC was assessed via TUNEL staining (Fig. 5A and C). At $24 \mathrm{~h}$ post-surgery, the TUNEL-positive cell number was greater in the SAH group compared with the sham group $(\mathrm{P}<0.05$; Fig. $5 \mathrm{~A}$ and $\mathrm{C})$, and 


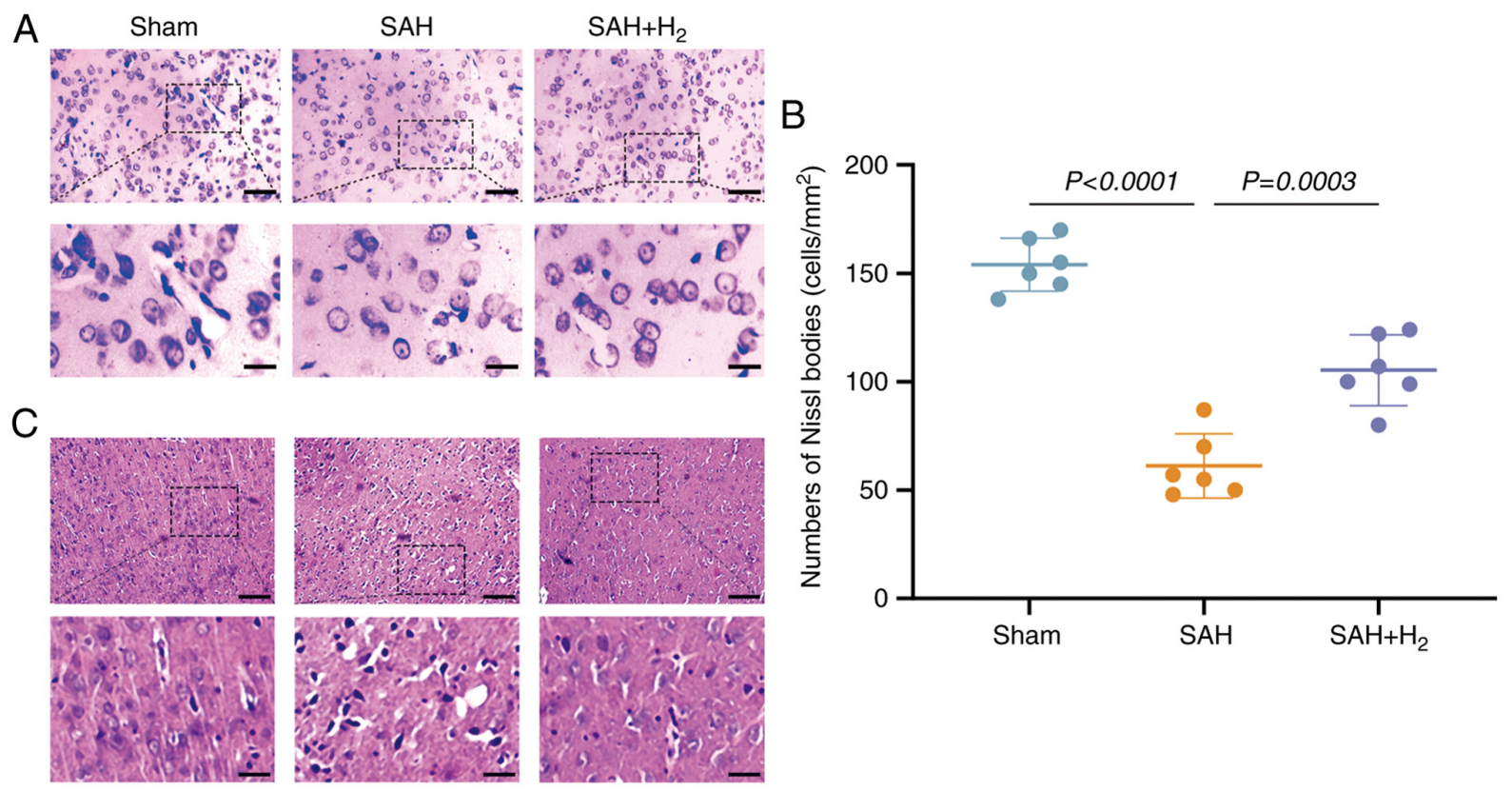

Figure 4. $\mathrm{H}_{2}$ post-conditioning reduces neuronal damage following SAH. (A) Representative Nissl staining of neurons in the vmPFC at 30 day post-SAH ( $\mathrm{n}=6$ ). Scale bar, 50 or $15 \mu \mathrm{m}$. (B) Numbers of Nissl bodies in the vmPFC, SAH and $\mathrm{SAH}+\mathrm{H}_{2}$ groups. $\mathrm{SAH}$ and $\mathrm{H}_{2}$ are as described previously. (C) Representative H\&E staining in the vmPFC at 30 days post-SAH. Scale bar, 50 or $15 \mu \mathrm{m}$. SAH, subarachnoid hemorrhage; vmPFC, ventromedial prefrontal cortex.

A
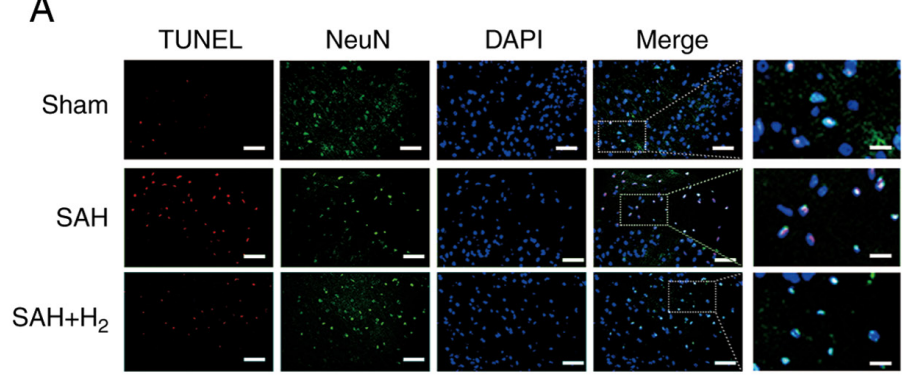

B

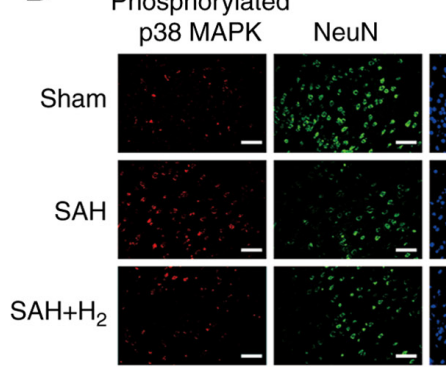

DAPI

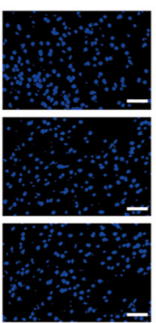

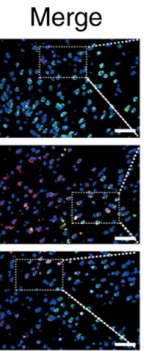

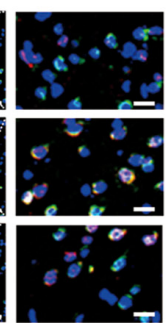

C

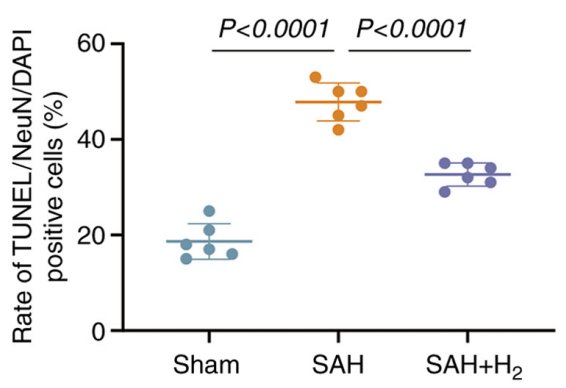

D

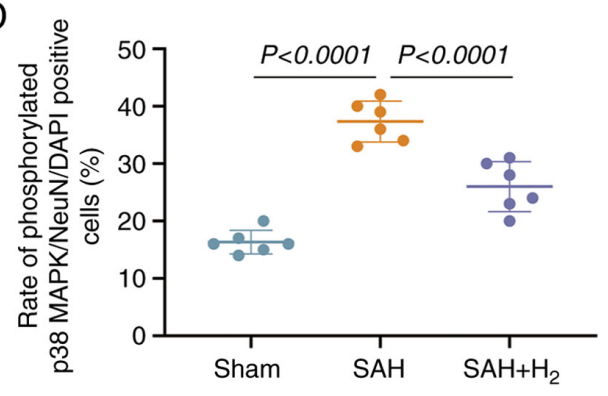

Figure 5. $\mathrm{H}_{2}$ post-conditioning reduces neuronal apoptosis and alters SAH-induced phosphorylated-p38 MAPK in neurons. (A) Representative photomicrographs of NeuN and TUNEL staining (NeuN, green; TUNEL, red; DAPI, blue), showing apoptotic neurons in the vmPFC on $24 \mathrm{~h}$ after SAH (n=6). Scale bar, 50 or $15 \mu \mathrm{m}$. (B) Representative photomicrographs of NeuN and phosphorylated p38 MAPK staining (NeuN, green; p38 MAPK, red; DAPI, blue), showing phosphorylated p38 MAPK-positive neurons in the vmPFC on $24 \mathrm{~h}$ after SAH (n=6). Scale bar, 50 or $15 \mu \mathrm{m}$. (C) Rate of apoptotic neurons in the vmPFC, induced by the indicated stimuli. (D) Rate of phosphorylated p38 MAPK-positive neurons in the vmPFC, induced by the indicated stimuli. SAH, subarachnoid hemorrhage; vmPFC, ventromedial prefrontal cortex; NeuN, neuronal nuclei.

this increase was partially reversed by $\mathrm{H}_{2}$ inhalation $(\mathrm{SAH}+$ $\mathrm{H}_{2}$ vs. SAH; $\mathrm{P}<0.05$; Fig. 5A and $\mathrm{C}$ ).

$\mathrm{H}_{2}$ inhalation inhibits the phosphorylation of p38 MAPK in neuronal cells. Immunostaining revealed the increased expression of p-p38 MAPK in NeuN-positive cells at $24 \mathrm{~h}$ post-SAH compared with the sham group $(\mathrm{P}<0.05$; Fig. $5 \mathrm{~B}$ and $\mathrm{D})$, a response significantly attenuated by $\mathrm{H}_{2}$ inhalation. Western blotting (Fig. 6A) also demonstrated the increased expression levels of p-p38 MAPK ( $\mathrm{P}<0.05$; Fig. 6B), p-JNK1/2 $(\mathrm{P}<0.05$; Fig. 6C), p-AKT ( $<<0.05$; Fig. 6D) and cleaved caspase-3 $(\mathrm{P}<0.05$; Fig. 6E) in the SAH group, as well as an elevated $\mathrm{Bax} / \mathrm{Bcl}-2$ ratio (vs. sham; $\mathrm{P}<0.05$; Fig. $6 \mathrm{~F}$ ). Again, each of these changes were reversed by $\mathrm{H}_{2}$ inhalation following $\mathrm{SAH}$. 

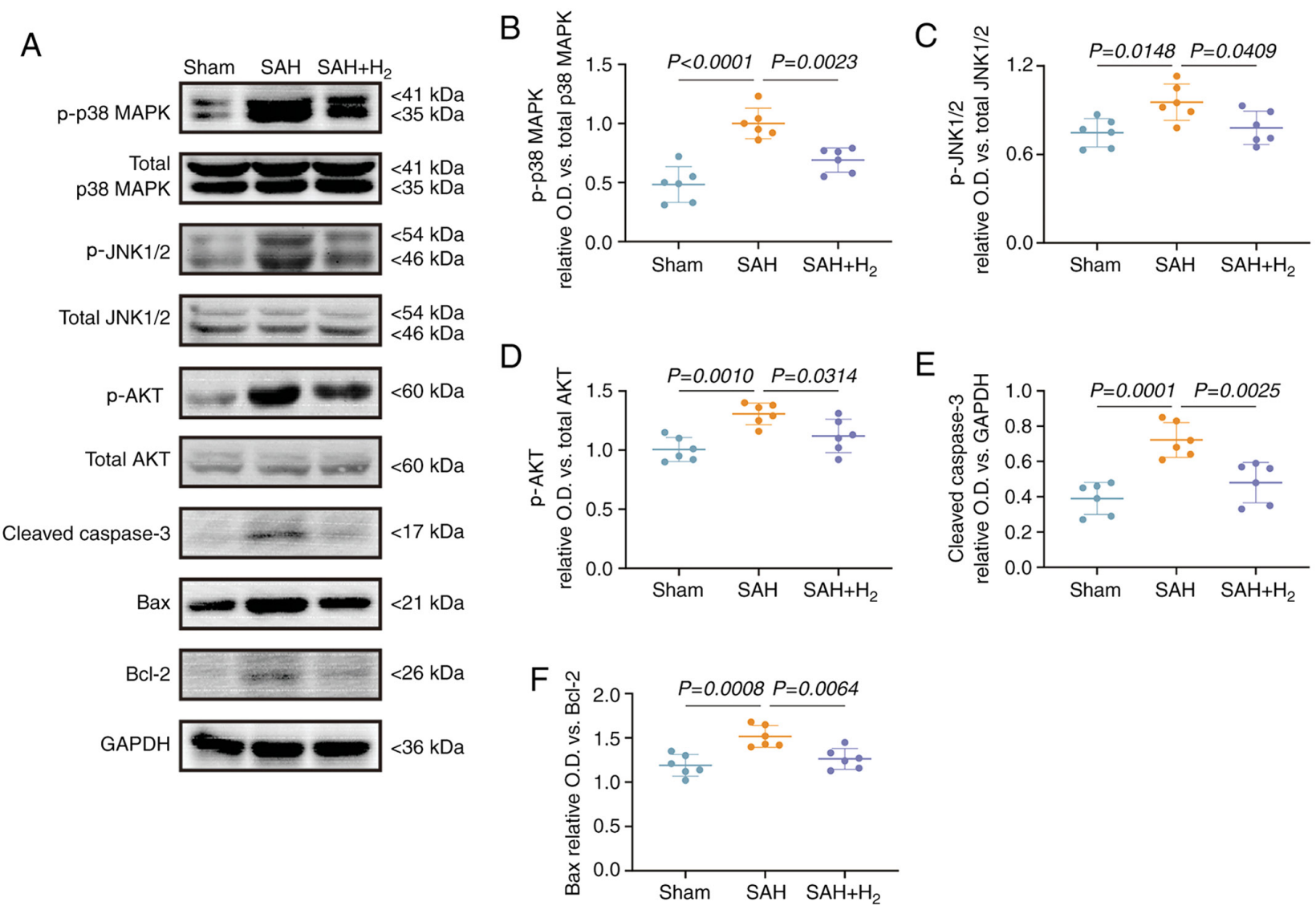

Figure 6. $\mathrm{H}_{2}$ post-conditioning inhibits the phosphorylation of p38 MAPK in neuronal cells. (A) Representative results of immunoblotting of p-p38 MAPK, total p38 MAPK, p-JNK1/2, total JNK1/2, p-AKT, total AKT (a second band of total Akt may be a non-specificity band), cleaved caspase-3, Bax and Bcl-2 in the vmPFC at $24 \mathrm{~h}$ post-SAH (n=6). (B) The ratio of p-p38 MAPK/total p38 MAPK. (C) The ratio of p-JNK1/2/total JNK1/2. (D) The ratio of p-AKT/total AKT. (E) The protein relative expression level of cleaved caspase-3. (F) The ratio of Bax/Bcl-2. SAH and $\mathrm{H}_{2}$ are as described previously. SAH, subarachnoid hemorrhage; p-, phosphorylated; O.D., optical density.

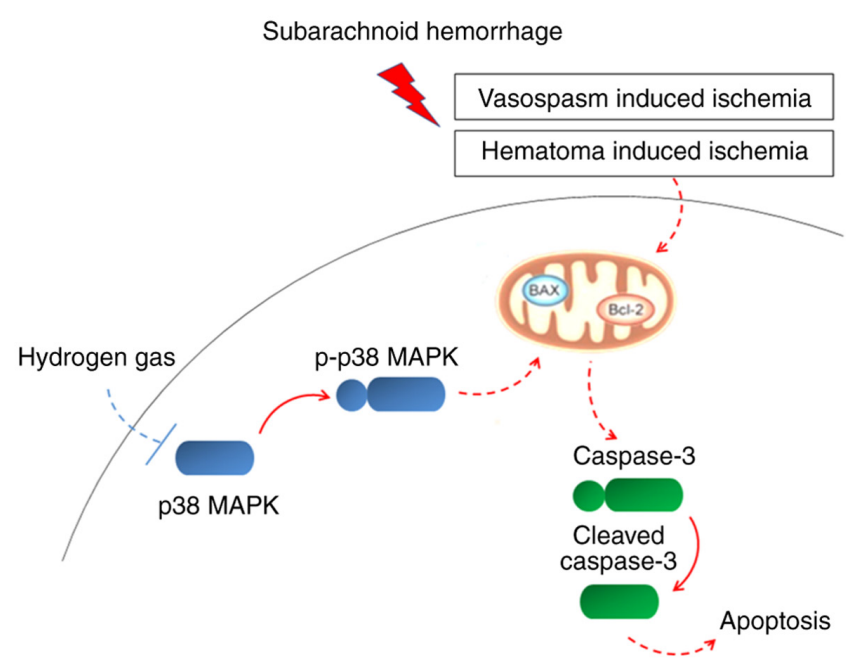

Figure 7. Schematic diagram of the signal pathway. A proposed schematic summarizing the observations following hydrogen gas post-conditioning and subsequent anti-apoptosis against $\mathrm{SAH}$ via the inhibition of phosphorylated p38 MAPK. SAH, subarachnoid hemorrhage; p-, phosphorylated.

\section{Discussion}

In this current study, it was demonstrated that relatively brief $\mathrm{H}_{2}$ inhalation following experimental SAH could mitigate metabolic disruption, long-term recognition memory impairment, anxiety-like behaviors and neuronal apoptosis in PFC, possibly by inhibiting p38 MAPK activity (Fig. 7).

Previous studies have reported that a single and double blood injection into cisterna magna, as well as endovascular perforation, can be used to establish a rodent model of SAH $(35,36)$. Compared with injections of arterial blood solvate into the cisterna magna, a rat model of endovascular perforation was performed in this current study due to a good consistency with the pathophysiological process of SAH (37). Neurological damage following SAH may result in cognitive decline and psychiatric disorders, such as anxiety and depression, which are major obstacles to rehabilitation and recovery (4). Previous studies have shown that rodent models of SAH were impaired in spatial memory as evidenced by longer escape latencies and swim paths in the Morris water maze test (38) and exhibited long-lasting state anxiety $(39,40)$. Considering motor deficits post-SAH, the present study assessed cognitive dysfunction and anxiety-like behavior as long-term behavioral changes at 30 days post-SAH. The current object recognition and OFT results were consistent with these previous studies $(41,42)$ and underscore the utility of this rodent model for investigation of SAH pathogenesis, behavioral impairments and potential treatment strategies. Furthermore, neuronal damage in the vmPFC is strongly associated with cognitive dysfunction and emotional changes following SAH $(43,44)$, and the present 
study identified significant neurocellular abnormalities in this region. While there were no significant differences in vmPFC SSI between sham and SAH groups, the NAA signal, a general marker of neuronal integrity and viability, was decreased after SAH exposure. The NAA pool is considered to act as a reservoir for glutamate synthesis that allows the brain to maintain lower glutamate concentrations and thereby reduce the risk of excitotoxicity (29). Collectively, these results suggested that long-term cognitive dysfunction and emotional changes induced by $\mathrm{SAH}$ may be associated with neuronal death in the vmPFC.

Recently, $\mathrm{H}_{2}$ administration was reported to attenuate neuronal injury in models of ischemia/reperfusion injury and hemorrhage-associated stoke $(45,46)$. In the current study, $2.9 \% \mathrm{H}_{2}$ treatment for $2 \mathrm{~h}$ after $\mathrm{SAH}$, chosen based on previous studies $(47,48)$ and our preliminary experiments, ameliorated cognitive dysfunction and anxiety-like behavior, as well as increased the number of Nissl bodies and elevated the NAA/Cr ratio. Interestingly, a previous study reported that $\mathrm{H}_{2}$ only improves neuronal apoptosis at $24 \mathrm{~h}$ after SAH, but not $48 \mathrm{~h}$. However, the present study found significant improvements in neuronal apoptosis in the vmPFC, which was different from previous studies $(10,25)$. As indicated by a previous study (49), 7 day post-SAH was the most severe period of cerebral vasospasm after SAH. In the present study, it was noted that there was no change of cerebral blood flow indicated by CBF-ASL after $\mathrm{H}_{2}$ administration 7 day post-SAH, which was consistent with previous study (50). It has been shown that apoptosis is a form of programmed cell death dependent on activation of caspase- 3 and is regulated by a relative balance between pro- and anti-apoptotic factors, such as Bax and Bcl-2 (51). The decrease in the apoptotic rate following $\mathrm{H}_{2}$ inhalation was associated with reduced expression of cleaved (active) caspase- 3 and Bax, and upregulated expression of Bcl-2, as measured via western blotting, which was consistent with a widespread reduction in apoptotic rate throughout the vmPFC. Thus, the present study revealed that $\mathrm{H}_{2}$ prevented long-term cognitive and emotional dysfunction following $\mathrm{SAH}$ by preventing neuronal apoptosis in the vmPFC, but not inhibiting cerebral vasospasm.

Activation of the p38 MAPK signal pathway can trigger apoptosis via a variety of downstream pathways, such as p53 phosphorylation (52), activation of caspase cascades and inhibition of anti-apoptotic proteins, such as Bcl-2 (53). The phosphorylation of p38 MAPK was reported to accelerate caspase- 3 cleavage and increase the $\mathrm{Bax} / \mathrm{Bcl}-2$ ratio in a model of traumatic brain injury (54). The present study demonstrated that $\mathrm{H}_{2}$ could reverse the increase in p-p38 MAPK expression by vmPFC neurons. Moreover, it was found that $\mathrm{H}_{2}$ downregulated the expression levels of $\mathrm{p}-\mathrm{JNK} 1 / 2$ and $\mathrm{p}-\mathrm{AKT}$ in the vmPFC. Previous studies also suggested that inhibition of JNK1/2 and AKT activations may be involved in the anti-apoptotic ability of $\mathrm{H}_{2}(55,56)$. Thus, besides JNK1/2 and AKT signaling, inhibition of p38 MAPK signaling is also a plausible mechanism for $\mathrm{H}_{2}$-dependent neuroprotection following SAH. The molecular mechanisms for this effect, including the contributions of antioxidant activity, require further study. Collectively, the current data indicated that single administration of $\mathrm{H}_{2}$ could suppress neuronal apoptosis via inhibition of $\mathrm{p} 38$ MAPK signaling.
Limitations of the present study include the single administration protocol and focus on the vmPFC. Future studies are required to define additional efficacious administration regimens, to determine whether later post-insult administration is also effective, as this has significant implications for clinical treatment, and to examine if $\mathrm{H}_{2}$ is neuroprotective in other regions associated with cognition and emotion, such as the hippocampus and amygdala. In addition, the current study did not examine the expression levels of other MAPK-related signaling factors, such as MAPKKK, MKK3/6 and apoptosis signal-regulating kinase 1 , to define a more precise pathway between p38 MAPK activation and apoptosis. The therapeutic effects of repeated $\mathrm{H}_{2}$ administration post-SAH should be also investigated.

In conclusion, $\mathrm{H}_{2}$ administered $2 \mathrm{~h}$ after experimental $\mathrm{SAH}$ mitigates cognitive dysfunction, anxiety-like behavior and neuronal apoptosis in the PFC, possibly by inhibiting the p38 MAPK signaling pathway.

\section{Acknowledgements}

Not applicable.

\section{Funding}

The present study was supported by the Science and Technology Plan of Cangzhou City (grant no. 172302117).

\section{Availability of data and materials}

The datasets used and/or analyzed during the present study are available from the corresponding author on reasonable request.

\section{Authors' contributions}

Design of the study: JHS. Editing the manuscript: JHS and TPS. Statistical analysis: JHS. Experiments and data collection: JHS, HYJ, TPS, ZBL and YPZ. JHS and HYJ confirm the authenticity of all the raw data. All authors read and approved the final manuscript.

\section{Ethics approval and consent to participate}

All study procedures and animal care protocols were approved by the Animal Review Board of Cangzhou Central Hospital (Cangzhou, China) and conformed to National Institutes of Health guidelines.

\section{Patient consent for publication}

Not applicable.

\section{Competing interests}

The authors declare that they have no competing interests.

\section{References}

1. Buunk AM, Spikman JM, Metzemaekers JDM, van Dijk JMC and Groen RJM: Return to work after subarachnoid hemorrhage: The influence of cognitive deficits. PLoS One 14: e0220972, 2019. 
2. Wong GK, Lam SW, Ngai K, Wong A, Siu D, Poon WS and Mok V; Cognitive Dysfunction after Aneurysmal Subarachnoid Hemorrhage Investigators: Cognitive domain deficits in patients with aneurysmal subarachnoid haemorrhage at 1 year. J Neurol Neurosurg Psychiatry 84: 1054-1058, 2013.

3. Miller BA, Turan N, Chau M and Pradilla G: Inflammation, vasospasm, and brain injury after subarachnoid hemorrhage. Biomed Res Int 2014: 384342, 2014.

4. Tang WK, Wang L, Kwok Chu Wong G, Ungvari GS, Yasuno F, Tsoi KKF and Kim JS: Depression after subarachnoid hemorrhage: A systematic review. J Stroke 22: 11-28, 2020.

5. Mo J, Enkhjargal B, Travis ZD, Zhou K, Wu P, Zhang G, Zhu Q, Zhang T, Peng J, Xu W, et al: AVE 0991 attenuates oxidative stress and neuronal apoptosis via Mas/PKA/CREB/UCP-2 pathway after subarachnoid hemorrhage in rats. Redox Biol 20: 75-86, 2019.

6. BlackmanRK,Crowe DA,DeNicola AL,Sakellaridi S,MacDonald AW III and Chafee MV: Monkey prefrontal neurons reflect logical operations for cognitive control in a variant of the $\mathrm{AX}$ continuous performance task (AX-CPT). J Neurosci 36: 4067-4079, 2016.

7. Zhang Y, Liu C, Wang J, Li Q, Ping H, Gao S and Wang P. MiR-299-5p regulates apoptosis through autophagy in neurons and ameliorates cognitive capacity in APPswe/PS1dE9 mice. Sci Rep 6: 24566, 2016.

8. Zare Z, Tehrani M, Zarbakhsh S, Farzadmanesh H, Shafia S, Abedinzade M, Ghanat A and Mohammadi M: Effects of paraoxon exposure on expression of apoptosis-related genes, neuronal survival, and astrocyte activation in rat prefrontal cortex. Neurotox Res 37: 356-365, 2020.

9. Zhang M, Shan H, Chang P, Wang T, Dong W, Chen X and Tao L: Hydrogen sulfide offers neuroprotection on traumatic brain injury in parallel with reduced apoptosis and autophagy in mice. PLoS One 9: e87241, 2014.

10. Choi KS, Kim HJ, Do SH, Hwang SJ and Yi HJ: Neuroprotective effects of hydrogen inhalation in an experimental rat intracerebral hemorrhage model. Brain Res Bull 142: 122-128, 2018.

11. Li S, Fujino M, Ichimaru N, Kurokawa R, Hirano S, Mou L, Takahara S, Takahara T and Li XK: Molecular hydrogen protects against ischemia-reperfusion injury in a mouse fatty liver mode via regulating HO-1 and Sirt1 expression. Sci Rep 8: 14019, 2018

12. Gao Y, Yang H, Chi J, Xu Q, Zhao L, Yang W, Liu W and Yang W: Hydrogen gas attenuates myocardial ischemia reperfusion injury independent of postconditioning in rats by attenuating endoplasmic reticulum stress-induced autophagy. Cell Physiol Biochem 43: 1503-1514, 2017.

13. Yan M, Yu Y, Mao X, Feng J, Wang Y, Chen H, Xie K and Yu Y: Hydrogen gas inhalation attenuates sepsis-induced liver injury in a FUNDC1-dependent manner. Int Immunopharmacol 71: 61-67, 2019.

14. Yonamine R, Satoh Y, Kodama M, Araki Y and Kazama T: Coadministration of hydrogen gas as part of the carrier gas mixture suppresses neuronal apoptosis and subsequent behavioral deficits caused by neonatal exposure to sevoflurane in mice. Anesthesiology 118: 105-113, 2013.

15. Bai X, Liu S, Yuan L, Xie Y, Li T, Wang L, Wang X, Zhang T, Qin S, Song G, et al: Hydrogen-rich saline mediates neuroprotection through the regulation of endoplasmic reticulum stress and autophagy under hypoxia-ischemia neonatal brain injury in mice. Brain Res 1646: 410-417, 2016.

16. Xie K, Zhang Y, Wang Y, Meng X, Wang Y, Yu Y and Chen H: Hydrogen attenuates sepsis-associated encephalopathy by NRF2 mediated NLRP3 pathway inactivation. Inflamm Res 69: 697-710, 2020

17. Wang P, Zhao M, Chen Z, Wu G, Fujino M, Zhang C, Zhou W, Zhao M, Hirano SI, Li XK and Zhao L: Hydrogen gas attenuates hypoxic-ischemic brain injury via regulation of the MAPK/HO-1/PGC-1a pathway in neonatal rats. Oxid Med Cell Longev 2020: 6978784, 2020.

18. Li D and Ai Y: Hydrogen saline suppresses neuronal cell apoptosis and inhibits the p38 mitogen-activated protein kinase-caspase-3 signaling pathway following cerebral ischemia-reperfusion injury. Mol Med Rep 16: 5321-5325, 2017.

19. Lee Y, Kim YJ, Kim MH and Kwak JM: MAPK cascades in guard cell signal transduction. Front Plant Sci 7: 80, 2016.

20. Zhang G, He J, Ye X, Zhu J, Hu X, Shen M, Ma Y, Mao Z, Song $\mathrm{H}$ and $\mathrm{Chen} \mathrm{F}$ : $\beta$-Thujaplicin induces autophagic cell death, apoptosis, and cell cycle arrest through ROS-mediated Akt and p38/ERK MAPK signaling in human hepatocellular carcinoma. Cell Death Dis 10: 255, 2019

21. Chen S, Ma Q, Krafft PR, Chen Y, Tang J, Zhang J and Zhang JH: $\mathrm{P} 2 \mathrm{X} 7$ receptor antagonism inhibits $\mathrm{p} 38$ mitogen-activated protein kinase activation and ameliorates neuronal apoptosis after subarachnoid hemorrhage in rats. Crit Care Med 41: e466-e474, 2013.
22. Li C, Liu Y, Tang P, Liu P, Hou C, Zhang X, Chen L, Zhang L and Gu C: Hydrogen sulfide prevents OGD/R-induced apoptosis by suppressing the phosphorylation of p38 and secretion of IL-6 in PC12 cells. Neuroreport 27: 230-234, 2016.

23. Sehba FA: The rat endovascular perforation model of subarachnoid hemorrhage. Acta Neurochir Suppl 120: 321-324, 2015.

24. Zhang CS, Han Q, Song ZW, Jia HY, Shao TP and Chen YP: Hydrogen gas post-conditioning attenuates early neuronal pyroptosis in a rat model of subarachnoid hemorrhage through the mitoK $_{\text {ATP }}$ signaling pathway. Exp Ther Med 22: 836, 2021.

25. Zhan Y, Chen C, Suzuki H, Hu Q, Zhi X and Zhang JH: Hydrogen gas ameliorates oxidative stress in early brain injury after subarachnoid hemorrhage in rats. Crit Care Med 40: 1291-1296, 2012.

26. Sugawara T, Ayer R, Jadhav V and Zhang JH: A new grading system evaluating bleeding scale in filament perforation subarachnoid hemorrhage rat model. J Neurosci Methods 167: 327-334, 2008.

27. Kooijman E, Nijboer CH, van Velthoven CT, Mol W, Dijkhuizen RM, Kesecioglu J and Heijnen CJ: Long-term functional consequences and ongoing cerebral inflammation after subarachnoid hemorrhage in the rat. PLoS One 9: e90584, 2014.

28. Marota JJ, Crosby G and Uhl GR: Selective effects of pentobarbital and halothane on c-fos and jun-B gene expression in rat brain. Anesthesiology 77: 365-371, 1992.

29. Fu L, Zhang DX, Zhang LM, Song YC, Liu FH, Li Y, Wang XP, Zheng WC, Wang XD, Gui CX, et al: Exogenous carbon monoxide protects against mitochondrial DNA-induced hippocampal pyroptosis in a model of hemorrhagic shock and resuscitation. Int J Mol Med 45: 1176-1186, 2020.

30. Oh HM, Lee JS, Kim SW, Oh YT, Kim WY, Lee SB, Cho YR, Jeon YJ, Cho JH and Son CG: Uwhangchungsimwon, a standardized herbal drug, exerts an anti-depressive effect in a social isolation stress-induced mouse model. Front Pharmacol 10: 1674, 2020.

31. Carriel V, Campos A, Alaminos M, Raimondo S and Geuna S: Staining methods for normal and regenerative myelin in the nervous system. Methods Mol Biol 1560: 207-218, 2017.

32. Cohen SJ and Stackman RW Jr: Assessing rodent hippocampal involvement in the novel object recognition task. A review. Behav Brain Res 285: 105-117, 2015

33. Vuralli D, Wattiez AS, Russo AF and Bolay H: Behavioral and cognitive animal models in headache research. J Headache Pain 20: 11, 2019.

34. Kangas BD and Bergman J: Touchscreen technology in the study of cognition-related behavior. Behav Pharmacol 28: 623-629, 2017.

35. Ostrowski RP, Colohan AR and Zhang JH: Neuroprotective effect of hyperbaric oxygen in a rat model of subarachnoid hemorrhage. Acta Neurochir Suppl 96: 188-193, 2006.

36. Güresir E, Schuss P, Borger V and Vatter H: Rat cisterna magna double-injection model of subarachnoid hemorrhage-background, advantages/limitations, technical considerations, modifications, and outcome measures. Acta Neurochir Suppl 120: 325-329, 2015.

37. Okada T, Enkhjargal B, Travis ZD, Ocak U, Tang J, Suzuki H and Zhang JH: FGF-2 attenuates neuronal apoptosis via FGFR3/PI3k/Akt signaling pathway after subarachnoid hemorrhage. Mol Neurobiol 56: 8203-8219, 2019.

38. Kumagai K, Tomiyama A, Takeuchi S, Otani N, Fujita M, Fujii K, Wada K and Mori K: New endovascular perforation subarachnoid hemorrhage model for investigating the mechanisms of delayed brain injury. J Neurosurg: Nov 22, 2019 (Epub ahead of print). doi: 10.3171/2019.9.JNS191934.

39. Shen H, Chen Z, Wang Y, Gao A, Li H, Cui Y, Zhang L, Xu X, Wang $Z$ and Chen G: Role of neurexin- $1 \beta$ and neuroligin-1 in cognitive dysfunction after subarachnoid hemorrhage in rats. Stroke 46: 2607-2615, 2015

40. Persson HC, Törnbom M, Winsö O and Sunnerhagen KS: Symptoms and consequences of subarachnoid haemorrhage after 7 years. Acta Neurol Scand 140: 429-434, 2019.

41. Nanegrungsunk D, Ragozzino ME, Xu HL, Haselton KJ and Paisansathan C: Subarachnoid hemorrhage in C57BL/6J mice increases motor stereotypies and compulsive-like behaviors. Neurol Res 43: 239-251, 2021

42. Donatti AF, Soriano RN, Leite-Panissi CR, Branco LG and de Souza AS: Anxiolytic-like effect of hydrogen sulfide $\left(\mathrm{H}_{2} \mathrm{~S}\right)$ in rats exposed and re-exposed to the elevated plus-maze and open field tests. Neurosci Lett 642: 77-85, 2017.

43. Boyko M, Azab AN, Kuts R, Gruenbaum BF, Gruenbaum SE, Melamed I, Brotfain E, Shapira Y, Cesnulis E and Zlotnik A: The neuro-behavioral profile in rats after subarachnoid hemorrhage. Brain Res 1491: 109-116, 2013 
44. Chen J, Liang J, Lin X, Zhang Y, Zhang Y, Lu L and Shi J: Sleep deprivation promotes habitual control over goal-directed control: Behavioral and neuroimaging evidence. J Neurosci 37: 11979-11992, 2017.

45. Henricks AM, Berger AL, Lugo JM, Baxter-Potter LN Bieniasz KV, Petrie G, Sticht MA, Hill MN and McLaughlin RJ: Sex- and hormone-dependent alterations in alcohol withdrawal-induced anxiety and corticolimbic endocannabinoid signaling. Neuropharmacology 124: 121-133, 2017.

46. Chen L, Chao Y, Cheng P, Li N, Zheng H and Yang Y: UPLCQTOF/MS-based metabolomics reveals the protective mechanism of hydrogen on mice with ischemic stroke. Neurochem Res 44: 1950-1963, 2019

47. Chen CH, Manaenko A, Zhan Y, Liu WW, Ostrowki RP, Tang J and Zhang JH: Hydrogen gas reduced acute hyperglycemia-enhanced hemorrhagic transformation in a focal ischemia rat model. Neuroscience 169: 402-414, 2010.

48. Huang L, Applegate RL II, Applegate PM, Boling W and Zhang JH: Inhalation of high concentration hydrogen gas improves short-term outcomes in a rat model of asphyxia induced-cardiac arrest. Med Gas Res 8: 73-78, 2018.

49. Xu H, Changyaleket B, Valyi-Nagy T, Dull RO, Pelligrino DA, Schwartz DE and Chong ZZ: The role of HMGB1 in pial arteriole dilating reactivity following subarachnoid hemorrhage in rats. J Vasc Res 53: 349-357, 2016.

50. Kumagai K, Toyooka T, Takeuchi S, Otani N, Wada K, Tomiyama A and Mori K: Hydrogen gas inhalation improves delayed brain injury by alleviating early brain injury after experimental subarachnoid hemorrhage. Sci Rep 10: 12319, 2020.

51. Zhang Y, Yang X, Ge X and Zhang F: Puerarin attenuates neurological deficits via Bcl-2/Bax/cleaved caspase-3 and Sirt3/SOD2 apoptotic pathways in subarachnoid hemorrhage mice. Biomed Pharmacother 109: 726-733, 2019.
52. Park SH, Seong MA and Lee HY: p38 MAPK-induced MDM2 degradation confers paclitaxel resistance through p53-mediated regulation of EGFR in human lung cancer cells. Oncotarget 7: 8184-8199, 2016.

53. Yang JS, Lin RC, Hsieh YH, Wu HH, Li GC, Lin YC, Yang SF and $\mathrm{Lu} \mathrm{KH}$ : CLEFMA activates the extrinsic and intrinsic apoptotic processes through JNK1/2 and p38 pathways in human osteosarcoma cells. Molecules 24: 3280, 2019.

54. Tan Z, Chen L, Ren Y, Jiang X and Gao W: Neuroprotective effects of FK866 against traumatic brain injury: Involvement of p38/ERK pathway. Ann Clin Transl Neurol 7: 742-756, 2020.

55. Qiu X, Li H, Tang H, Jin Y, Li W, YuSun, PingFeng, Sun X and Xia Z: Hydrogen inhalation ameliorates lipopolysaccharide-induced acute lung injury in mice. Int Immunopharmacol 11: 2130-2137, 2011.

56. Wang Y, Wang L, Hu T, Wang F, Han Z, Yin Z, Ge X, Xie K and Lei P: Hydrogen improves cell viability partly through inhibition of autophagy and activation of PI3K/Akt/GSK3 $\beta$ signal pathway in a microvascular endothelial cell model of traumatic brain injury. Neurol Res 42: 487-496, 2020.

This work is licensed under a Creative Commons Attribution-NonCommercial-NoDerivatives 4.0 International (CC BY-NC-ND 4.0) License. 